\title{
DISTRIBUCIÓN ONLINE DE CONTENIDOS AUDIOVISUALES: ANÁLISIS DE 3 MODELOS DE NEGOCIO
}

\author{
Jessica Izquierdo-Castillo
}

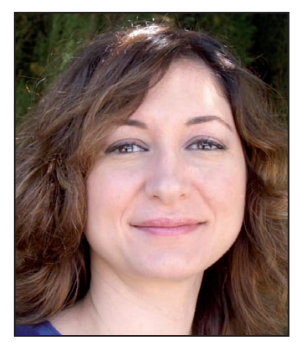

Jessica Izquierdo-Castillo es profesora en la Universitat Jaume I (UJI) de Castellón. Es doctora en comunicación audiovisual y licenciada en publicidad y RRPP por la UJl, y en comunicación audiovisual por la Universitat de València. Es miembro de los grupos de investigación Investigación en tecnologías aplicadas a la comunicación audiovisual (Itaca) y Periodismo, comunicación y poder, en el Departamento de Ciencias de la Comunicación de la UJI.

Universitat Jaume I de Castellón Dpto. Ciencias de la Comunicación Av. Vicente Sos Baynat, s/n. 12071 Castellón, España jizquier@uji.es

\section{Resumen}

La convergencia mediática está transformando el modelo de negocio audiovisual. El consumo de contenidos cinematográficos y televisivos en internet impone la ruptura de la cadena de valor tradicional. Se analizan las bases de los modelos de negocio online para la distribución y el consumo de estos contenidos. Se toman las fuentes de financiación y su reconfiguración respecto al modelo tradicional: tarifa plana, pago por visión y publicidad. Se estudian tres casos vinculados con cada una de las fórmulas de monetización: Netflix, iTunes y Hulu. Finalmente, se ofrece una propuesta de modelo de negocio.

\section{Palabras clave}

Distribución, Online, Internet, Cine, Televisión, Multimedia, Contenidos, Audiovisual.

Title: Online distribution of media content: analysis of 3 business models

\begin{abstract}
Media convergence is transforming the media business model. Film and television consumption over the internet force the breakdown of the traditional value chain. This paper aims to study the basics of online business models created to distribute and consume media content. This study considers funding sources and the reconfiguration of traditional model: flat rate, pay per view and advertising. The methodology is based on three case studies, connected to each of the funding formulas: Netflix, iTunes and Hulu. Results are intended to provide a business model proposal.
\end{abstract}

\section{Keywords}

Distribution, Online, Internet, Film, Television, Multimedia, Content, Media.

Izquierdo-Castillo, Jessica. "Distribución online de contenidos audiovisuales: análisis de 3 modelos de negocio". El profesional de la información, 2012, julio-agosto, v. 21, n. 4, pp. 385-390.

\section{Introducción}

La distribución online de contenidos plantea un escenario de gran potencial para la comunicación digital. La convergencia mediática reúne en un mismo contexto a los actores comunicativos tradicionales, mientras provoca la emergencia constante de actores nativos digitales (pure players). En lo referente a los productos audiovisuales, internet plantea un desafío para la consolidación de modelos de negocio compatibles con la cultura de consumo del nuevo espectador-internauta.

Las transformaciones en el flujo de comercialización de los productos audiovisuales tienen implicaciones en tres niveles:
1) se modifica la cadena de valor tradicional (Álvarez-Monzoncillo, 2011);

2) se ven afectados los hábitos de consumo del espectador, con el que se establece una mutua influencia que repercute en la búsqueda de modelos rentables para ambos;

3) se reconfigura la propia estructura del sistema audiovisual, principalmente en producción y distribución.

Además aparece un nuevo actor que acumula protagonismo día tras día: los operadores de telecomunicación, encargados de vehicular el contenido.

La conjunción de los tres niveles afectados reclama el asen- 
tamiento de fórmulas de monetización válidas y estables, que permitan a la industria evolucionar en términos de rentabilidad. Los actores tradicionales comienzan a asumir, a su pesar, la no inagotabilidad del modelo tradicional, que se destruye en el contexto online. Los principales proveedores de contenidos tradicionales, las majors ${ }^{1}$ (MGM, Paramount, Sony, Warner Bros...), han claudicado ante dos factores incontestables: por un lado, la evidencia de las darknets ${ }^{2}$ (Biddle et al., 2002) y el consumo paralelo de sus productos; por otro, la presión de los distribuidores online, que demandan mayor implicación y un catálogo con oferta más atractiva (Papies; Clement, 2008). La aventura digital supone la elaboración de modelos de negocio que moneticen el contenido (Álvarez et al., 2011). Sin embargo, a pesar de los cambios, en internet se observan "las mismas posiciones de dominio y barreras de entrada" que en el escenario convencional (Álvarez-Monzoncillo, 2011, p. 3). Como resultado se perfilan estructuras diversas, marcadas por un lado por la entrada de nuevos intermediarios que agrupan contenidos sin producirlos, y negocian con los agentes tradicionales para la elaboración del catálogo ofertado. Y por otro lado, los operadores de telecomunicación que van adquiriendo protagonismo a medida que se consolidan como la infraestructura necesaria para canalizar el contenido.

\section{Digitalización en el origen de la distribución online}

Trataremos ahora de las plataformas de distribución online de contenidos audiovisuales, principalmente cine y televisión. Estas dos industrias han permanecido ancladas al modelo tradicional, mientras que otras ramas del entretenimiento (música) y de la información (prensa) se han visto forzadas a su adaptación al medio online. La distribución de películas en internet surgió asociada al proceso de digitalización del sector, lo que amenazó las bases sobre las que se asienta la hegemonía de las majors norteamericanas. Estas distribuidoras, dueñas de los principales mercados durante años, se esforzaron por ralentizar el proceso con la excusa de los estándares y la piratería (Izquierdo, 2010). Pero la tecnología digital no era el fin, sino el medio para que el cine (y la televisión) penetraran en el actual universo de tecnologías convergentes.

En el contexto digital la Red presiona sobre la industria albergando y elaborando formas de consumo fuertemente influenciadas por la cultura de la gratuidad y el acceso instantáneo. Sin embargo, internet no excluye el negocio y esta premisa ha quedado validada con la reconfiguración del modelo de la música, que ahora intentan seguir los productos informativos, en especial la prensa, muy afectada por la digitalización (Casero-Ripollés, 2010). En este sentido, la teoría de la long-tai ${ }^{3}$ defiende el potencial de la
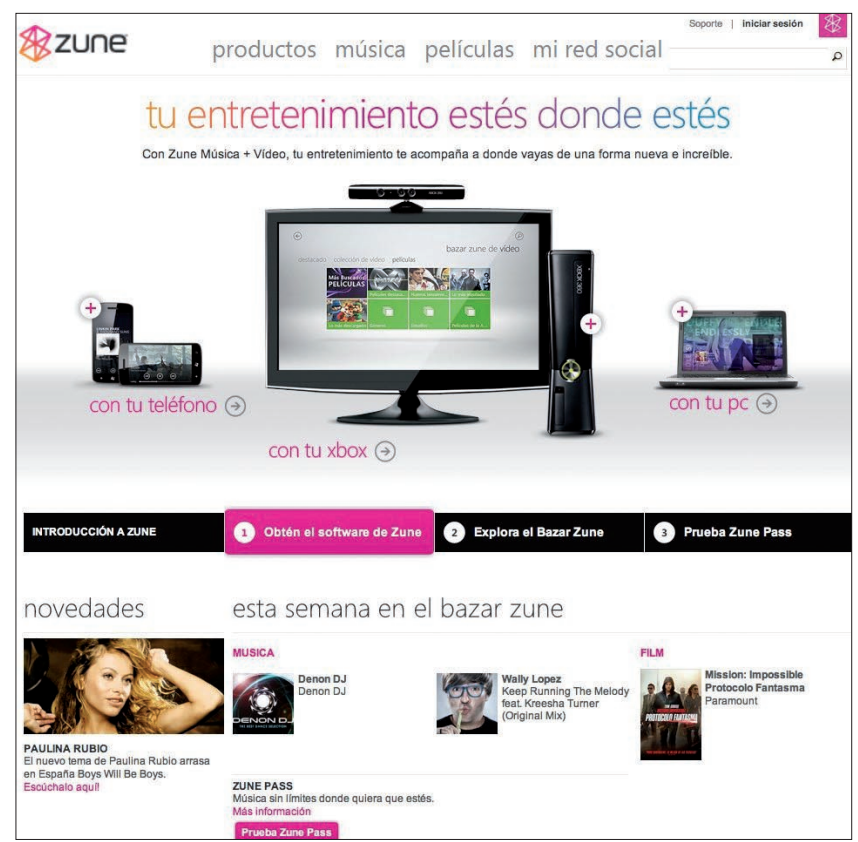

http://www.zune.net/es-es

Red para el desarrollo de mercado (Anderson, 2007; Elberse, 2008), pues puede distribuir una variada gama de productos (películas) destinados a una amplia, numerosa y dispersa demanda. Así, la reducción de costes de distribución debida a la Red (Miguel-de-Bustos, 2007) sustituye -o complementa- el modelo hegemónico mantenido durante años por las majors (Augros, 2000), basado en la distribución, intensiva en el tiempo y extensiva en la cobertura de grandes superproducciones (Miller, 2005; Wasko, 2003; Augros, 2000; Gomery, 1991). La Red permite estimular la creación y venta de productos dirigidos a públicos minoritarios, que, considerados de forma agregada, constituyen una demanda importante. De esta forma, Internet contribuye a aumentar la oferta y variedad de títulos, así como a flexibilizar la en-

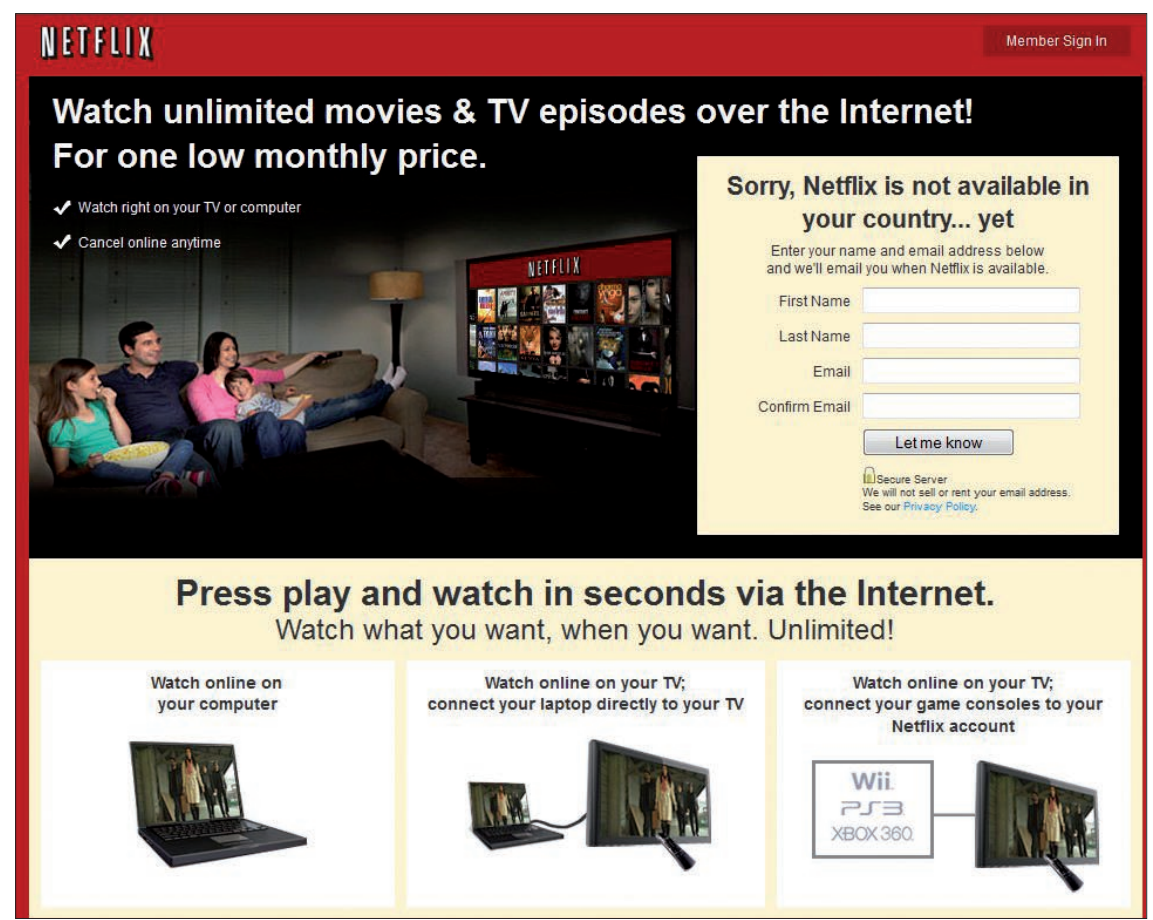

https://signup.netflix.com/global 
trada de contenidos alternativos y de nuevos agentes intermediarios (Casero-Ripollés, 2008). Por lo tanto, el cine se suma a la experimentación de las alternativas online, que le facilitan el acceso transnacional de las audiencias (Ryan; Hearn, 2010). Se incrementan las opciones de consumo en diversas plataformas que combinan las películas con el resto de oferta audiovisual.

La distribución online no sólo transforma el modelo de negocio, sino también, y como consecuencia, el consumo de películas. Este proceso de digitalización se ha acelerado por tres factores:

- aumento del ancho de banda y disponibilidad de monitores de alta definición para ordenador;

- incremento de plataformas con capacidad de reproducción de vídeo (móviles, tabletas, videoconsolas, etc.); y

- auge de modelos de negocio en internet.

Todo ello ha contribuido a la reestructuración del sector cinematográfico, disrumpiendo el modelo tradicional (Cunningham; Silver; McDonnell, 2010).

\section{Los tres modelos principales de la distribución online de contenido audiovisual}

Al principio el cine se introdujo en internet simplemente en forma de anuncios, como escaparate de exposición de películas para estimular el mercado de venta y alquiler videográfico. A partir de ahí, la evolución tecnológica reclamaba la digitalización de los contenidos. Sin embargo, las majors presentaron reticencias a esta digitalización, ya que amenazaba su mercado post-sala (DVD y televisión) (Papies; Clement, 2008). No obstante, el potencial comercial de in-

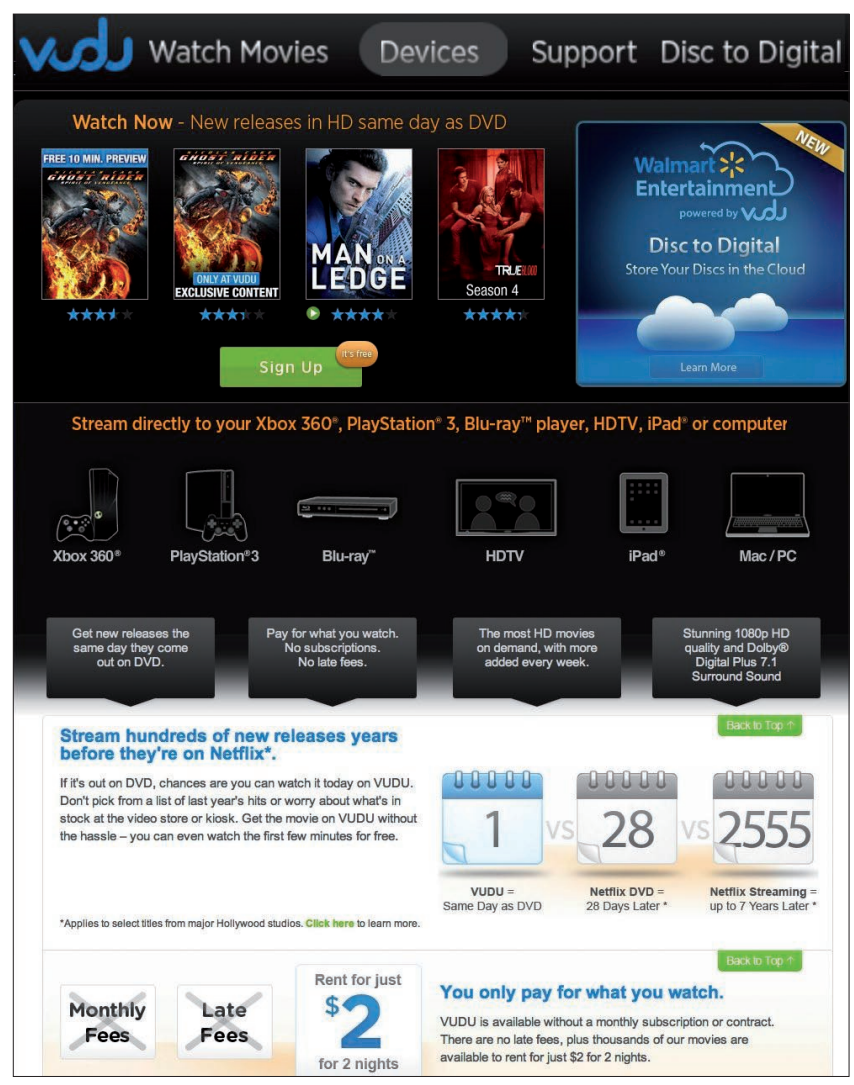

http://www.vudu.com ternet ha contribuido a la flexibilización del negocio (Hennig-Thurau et al., 2007), pero también a la destrucción de otros intermediarios (Miguel-de-Bustos, 2007). Como resultado, el mercado de la distribución online de contenido audiovisual (de cine y televisión, principalmente) se dibuja en torno a tres modelos diferenciados, que corresponden a tres fórmulas de financiación:

\section{Micropago}

Es el esquema de pago por unidad, ya sea en forma de vídeo bajo demanda (video on demand, o iVOD, siendo la i interactive o internet), entendido como el consumo en directo del contenido en el momento en que es solicitado por el público, o por medio de venta electrónica, en el que el público adquiere el contenido en propiedad. En este grupo destacan la plataforma de Apple: iTunes; el portal de distribución de Microsoft: VideoZune; y el gestionado por los supermercados Wal-Mart: Vudu. El amplio catálogo de productos disponibles en la Apple Store sitúa a la compañía en el puesto de liderazgo de la venta electrónica estadounidense (electronic sell through, EST) ${ }^{4}$ y de vídeo bajo demanda online (iVOD), con una cuota de mercado del 64,5\% en 2010 (tabla 1). El modelo de iTunes se ve (re)forzado por la sujeción a plataformas propias de visionado (iPod, iPhone, iPad) y por el desarrollo de la Apple TV.

\begin{tabular}{|c|l|c|c|c|}
\hline Posición & \multicolumn{1}{|c|}{ Compañía } & $\mathbf{2 0 0 9}$ & $\mathbf{2 0 1 0}$ & $\mathbf{2 0 1 1 *}^{*}$ \\
\hline 1 & iTunes (Apple) & 74,4 & 64,5 & 65,8 \\
\hline 2 & Zune (Microsoft) & 11,6 & 17,9 & 16,2 \\
\hline 3 & Vudu (Wal-Mart) & - & 1,0 & 5,3 \\
\hline 4 & PlaySation Store (Sony) $^{*}$ & 5,7 & 7,2 & 4,4 \\
\hline 5 & Amazon $^{*}$ & - & 4,0 & 4,2 \\
\hline
\end{tabular}

* Los datos de ese año pertenecen al informe de agosto 2011. Vudu y Amazon no aparecen en el informe de febrero, donde se recogen los datos de 2009. Fuente: IHS Screen Digest (febrero 2011)

Tabla 1. Cuota de mercado de la venta electrónica y el VOD por internet en EUA en el período 2009-2010 (en porcentaje)

El modelo de micropago, instaurado por iTunes, ha supuesto el principal motor de las ventas electrónicas de contenidos audiovisuales. De hecho, el consumo electrónico estadounidense creció un $60 \%$ en 2010 , lo que se debe, en gran medida, al mantenimiento del liderazgo de la tienda virtual de Apple.

Sin embargo, durante el último trienio la plataforma de $\mathrm{Mi}$ crosoft para la venta y alquiler de películas se consolidó en la segunda posición, aunque muy alejada del primer puesto. El lanzamiento de Kinect 3D, el dispositivo de control de movimiento para su videoconsola Xbox 360, contribuyó a reforzar su situación, con un aumento en los ingresos de la compañía en el cuarto trimestre de 2010. Sin embargo, el rival con mayor potencial de iTunes es una cadena de supermercados (Wal-Mart), que inició su negocio de distribución online a través de Vudu, favorecida por los acuerdos ventajosos firmados con las majors, a quienes repercute un montante de 3.500.000 millones de dólares en concepto de ventas de copias físicas en su red de tiendas (2010). Con estos acuerdos, Vudu se permite ofertar películas comerciales a precios inferiores a un dólar, lo que le otorga una gran ventaja sobre iTunes, donde el coste de alquiler es de 3-5 dóla- 


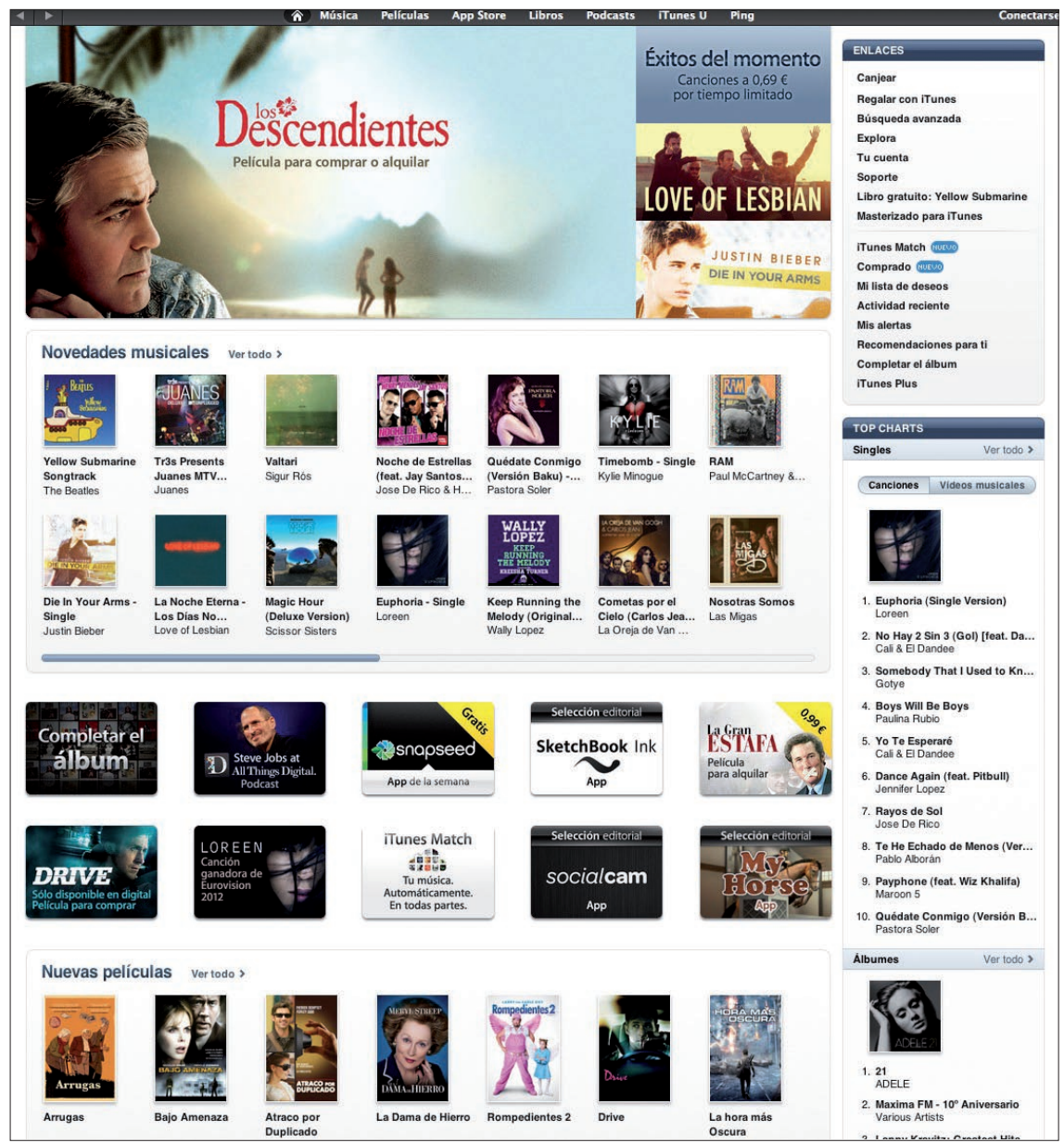

http://www.apple.com/itunes/charts/movies

res por película. Además Vudu se adapta a otros dispositivos de reproducción, mientras que iTunes sólo lo ofrece para los aparatos de Apple.

\section{Cuota mensual}

Sistema que permite el visionado ilimitado de contenido por una cantidad que oscila entre 7,99 y 9,99 dólares al mes. En este grupo destacan la plataforma nativa digital -pure player-Netflix, y la red de alquiler Blockbuster, en su versión telemática. Su principal aportación ha sido adaptar la evolución tecnológica a las demandas de entretenimiento audiovisual. Estos distribuidores se han ido adaptando a las posibilidades técnicas de la Red, comenzando con la distribución offline (alquiler de DVDs). Netflix comenzó con el negocio del alquiler de copias físicas en 1997 y en 2006-2010 incorporó el servicio de streaming, con unos resultados excelentes, ya que experimentó un crecimiento del 116\% ( $\mathrm{Ne}$ tflix, 2010; 2011). Sin embargo, durante el tercer trimestre de 2011 Netflix experimentó una pérdida de un millón de abonados (había comenzado el período con 20 millones de ellos). El principal motivo fue la escisión de los servicios de alquiler y streaming, de forma que la cuota de 9,99 dólares mensuales que pagaba un abonado por el acceso ilimitado al alquiler de DVDs y visionado de streaming, se convirtió en una cuota de 7,99 dólares por cada unidad de negocio. Este incremento del 37,5\% ahuyentó a los consumidores, que buscaron vías alternativas. En la actualidad el portal se centra en contenidos cinematográficos de alquiler, pero no oferta otros productos, como videojuegos, y tampoco incluye en su catálogo la venta de estrenos. Esta decisión le desplaza de la clasificación de los distribuidores online con mayor cuota de mercado (tabla 1), liderada por iTunes y su modelo de micropago. Para avanzar en la clasificación, Netflix está experimentando con otras opciones, como el iVOD y la adquisición en exclusiva de productos televisivos. Con esta idea, el portal pujó en 2011 por la serie digital HouseCards (David Fincher), arrebatándosela a la cadena de televisión norteamericana $H B O$.

\section{Modelo dependiente de la publi- cidad}

Propone fórmulas de acceso gratuitas sustentadas por los anunciantes. La gratuidad es un gran atractivo para el espectador, que encuentra una fórmula de acceso similar a la conocida en el escenario offline. Sin embargo, carece de aliciente para los operadores. De hecho, los datos apuntan a la inviabilidad de este modelo, pues la previsión de la publicidad online estima un $60 \%$ menos de crecimiento que la generada por los mismos programas distribuidos por televisión (Richmond, 2009).

La plataforma Hulu es un caso de referencia de pago por publicidad. Se trata de una iniciativa de NBC Universal y de News Corporation, con el soporte financiero de Providence Equito Partners. El éxito de Hulu quedó registrado cuando en 2008, un año después del inicio de su actividad, se convirtió en la segunda web de iVOD (Nielsen, 2009), superando a otros portales consolidados como Yahoo, Fox o Nickelodeon ${ }^{5}$. El sistema es gratuito para los consumidores, a quienes suministra contenidos en streaming de las principales operadoras de televisión. El catálogo recoge productos de las compañías fundadoras y de las que se han ido sumando con posterioridad (The Walt Disney Company se unió en 2009). Dispone de un portal de vídeo que distribuye contenidos televisivos y películas de las principales majors. Su flexibilidad le permite alojarse en plataformas de terceros, como Yahoo!, MSN, $A O L, I M D b$ y TV Guide, así como ubicar su reproductor en páginas personales de internautas, de forma similar a Youtube, pero con una perspectiva profesional (Artero, 2010). A pesar de los buenos resultados cosechados por el portal (40 millones de espectadores a finales de 2009, tan sólo un año después de su inicio, y 500 millones streams mensuales), este modelo reportó previsiones de pérdidas por valor de 33 millones de dólares (Walsh, 2009).

La dificultad de mantener la financiación exclusiva por publicidad plantea la inoperabilidad del modelo de acceso gratuito. De hecho, Hulu no descarta introducir otras formas de recaudación combinadas, con lo que intentaría evitar la paradoja de Youtube, que no encuentra cómo rentabilizar la gran cantidad de usuarios que tiene. En este sentido, Youtube se enfrenta a una situación marcada por el escaso 


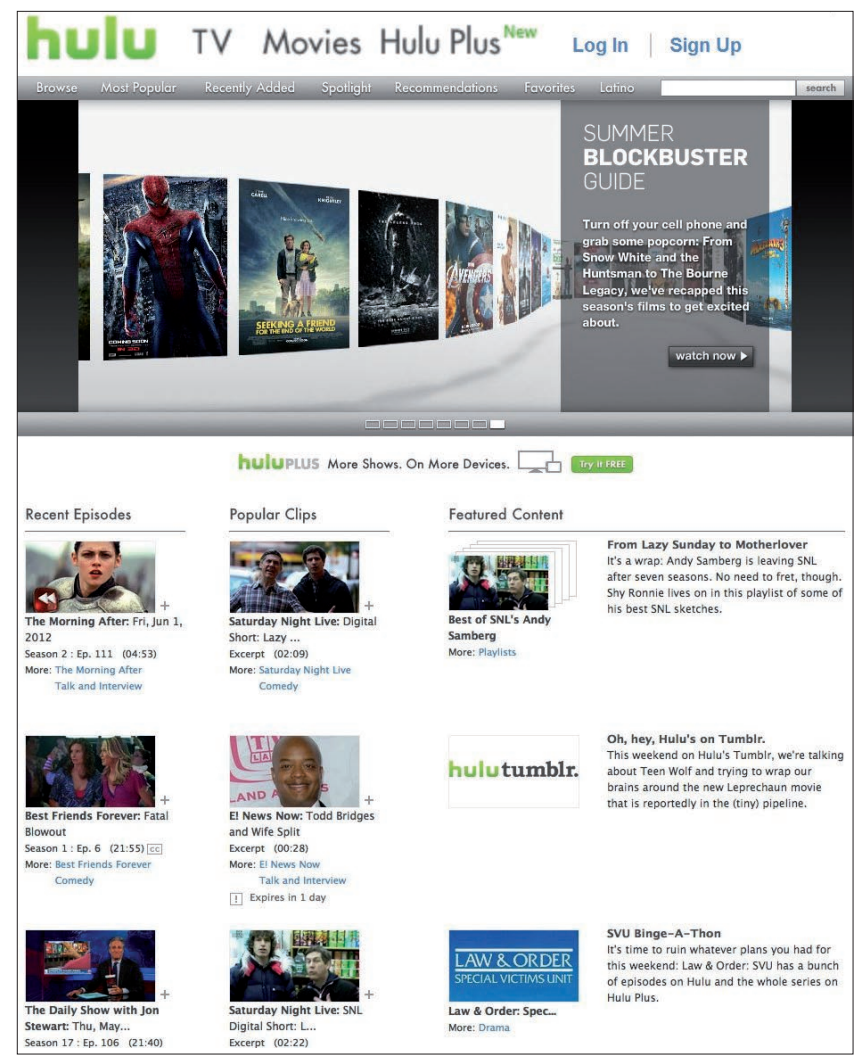

http://www.hulu.com

caudal de ingresos por publicidad (únicamente el $10 \%$ de los vídeos consultados inserta anuncios), lo que dificulta la amortización de equipos, la renovación de servidores y el mantenimiento del ancho de banda (Manjoo, 2009), una situación que pretende paliar con la introducción de películas y contenidos Premium (Parrick, 2009). De esta forma Youtube se pretende consolidar como la segunda fuerza en este modelo, ya que cuenta con los contenidos de las majors que han entrado en conflicto con Hulu.

El sistema de cuota mensual permite el visionado ilimitado de contenido por una cantidad que oscila entre 8 y 10 dólares al mes

\section{Conclusiones}

El estudio de las fuentes de financiación aplicadas en la distribución online de contenidos audiovisuales evidencia tres modelos: micropago, tarifa plana y publicidad. A pesar de la iniciativa original, ligada estrechamente a una única vía de ingresos, cada uno de los portales analizados presenta una combinación de las fórmulas a medida que aumenta su presencia en internet. Por otro lado, estas fuentes de financiación son adaptaciones de modos de pago utilizados en la comercialización tradicional, principalmente en el campo de las operadoras de televisión, que han ofrecido contenidos mediante cuotas de abonado (tarifa plana sujeta a una programación), el pago por unidad de producto (elegido de un catálogo limitado) y el acceso gratuito (televisión en abierto), donde la programación de cine ha significado su principal reclamo.
Los casos analizados en este estudio muestran una evolución que ayuda a plantear una propuesta de modelo de negocio para la distribución online, basado en tres puntos clave:

- adquisición de derechos de comercialización de un amplio catálogo, que contemple una gran diversidad de géneros, precios y público objetivo. Esto permitiría potenciar la producción, que no sólo beneficiaría al sector, sino que satisfaría al gran público que constituye la demanda de la larga cola, mientras que el contenido premium continuaría en manos de los actores hegemónicos (majors) que, no obstante, perderían la autonomía en el contexto de diversidad creativa y comercial;

- combinación de fórmulas de financiación, que ofrece mayor rentabilidad no sólo al distribuidor, sino también al consumidor;

- flexibilización de la reproducción, que permita el consumo en cualquier dispositivo móvil.

Los modelos basados en una única fuente de monetización han permitido a estas plataformas captar clientes. El grado de éxito que alcancen depende de la aplicación de estas claves $y$, sobre todo, del diseño que realicen de su oferta de contenidos.

\section{La dificultad de mantener la financiación exclusiva por publicidad plantea la inope- rabilidad del modelo de acceso gratuito}

\section{Notas}

1. La industria de producción y distribución de cine incluye cerca de 11.000 empresas con ingresos anuales de alrededor de $\$ 60.000$ millones. Las majors o principales empresas son Disney, Fox, MGM, Paramount, Sony Pictures, Universal y Warner Bros. La industria está muy concentrada: las 50 mayores empresas representan alrededor del $80 \%$ de los ingresos.

2. Una darknet o red oscura es una colección de tecnologías usadas para compartir contenidos digitales, tratando de ocultar la identidad de las entidades que intercambian dichos contenidos, es decir, persiguen el anonimato del origen y el destino cuando se produce la transferencia de información. Generalmente son invisibles a los motores de búsqueda.

3. Long tail o larga cola es un concepto introducido por Chris Anderson, según el cual en internet existen muchas posibilidades de hacer negocio aunque no se posea una web principal muy visitada. Inversamente: muchos pequeños clientes pueden dar lugar a un negocio fructífero.

4. Electronic sell-through (EST) [venta electrónica], es un método de distribución de medios por el cual los consumidores pagan una tarifa una única vez para descargar un archivo. A pesar de que se describe a menudo como una transacción que otorga al consumidor la "propiedad" del contenido, éste puede quedar inservible después de un período determinado y puede no ser visible en plataformas de 
la competencia. A veces se denomina "download-to-own" (DTO).

5. Nickelodeon, a menudo llamado simplemente Nick y originalmente llamado Pinwheel, es un canal infantil de Estados Unidos propiedad de Viacom. Está dirigido principalmente a los niños de escuela primaria y adolescentes jóvenes.

\section{Bibliografía}

Álvarez, Ignacio; Benamou, Jérémy; Fernández-Bosch, Jose-Manuel; Solé, Clara. España cONecta. Cómo transforma internet la economía española. Boston: The Boston Consulting Group, 2011.

http://www.espanaconecta.es/pdf/BCG_espanaconecta.pdf

Álvarez-Monzoncillo, José-María. La televisión etiquetada: nuevas audiencias, nuevos negocios. Madrid: Ariel, 2011. ISBN: 9788408098706

Anderson, Chris. La economía longtail. Barcelona: Urano, 2007. ISBN: 9788493464264

Artero, Juan P. “Online video business models: Youtube vs Hulu". Palabra clave, 2010, v. 13, n. 1, pp. 111-123. http://palabraclave.unisabana.edu.co/index.php/palabra clave/article/view/1637/2073

Augros, Joël. El dinero de Hollywood. Financiación, producción, distribución y nuevos mercados. Barcelona: Paidós, 2000. ISBN: 8449308488

Biddle, Peter; Engl, Paul; Peinado, Marcus; Willman, Bryan. "The darknet and the future of content distribution". En: ACM Workshop on digital rights management (18 Nov. 2002).

Casero-Ripollés, Andreu. "Prensa en internet: nuevos modelos de negocio en el escenario de la convergencia". El profesional de la información, 2010, v. 19, n. 6, pp. 595-601. http://eprints.rclis.org/bitstream/10760/15015/1/595-601. $p d f$

http://dx.doi.org/10.3145/epi.2010.nov.05

Casero-Ripollés, Andreu. “¿Larga cola en el cine? El impacto de la economía reticular en la industria cinematográfica". En: Pérez-Perucha, Julio; Gómez-Tarín, Francisco-Javier; Rubio-Alcover, Agustín (eds). Temperatura crítica: el cine español de los 60 y las rupturas de la modernidad. Castellón: Universitat Jaume I, 2008.

Cunningham, Stuart; Silver, Jon; McDonnell, John. "Rates of change: online distribution as disruptive technology in the film industry". Media international australia, incorporating culture \& policy, 2010, n. 136, pp.119-132.

http://eprints.qut.edu.au/39387

Elberse, Anita. "Should you invest in the long-tail?" Harvard business review, 2008, July.

Gomery, Douglas. Hollywood: el sistema de estudios. Madrid: Verdoux, 1991. ISBN: 8440492189

Henning-Thurau, Thorsten; Henning, Victor; Sattler, Henrik; Eggers, Felix; Houston, Mark B. "The last picture show?
Timing and order of movie distribution channels". Journal of marketing, 2007, v. 71, n. 4, pp. 63-83.

Izquierdo-Castillo, Jessica. El cine digital. La distribución y exhibición españolas ante el reto tecnológico. Madrid: Ediciones de las Ciencias Sociales, 2010. ISBN: 9788487510564

Jenkins, Henry. "Transmedia storytelling". Technology review. Cambridge: Massachusetts Institute of Technology (MIT), Jan. 2003.

http://www.technologyreview.com/biomedicine/13052

Manjoo, Farhad. "Do you think bandwidth grows on trees?" Slate technology, 2009, 14 April.

Miguel-de-Bustos, Juan-Carlos. "Cambios institucionales en las industrias culturales. Hacia una economía directa o reticular". Portal de la comunicación, 2007.

http://www.portalcomunicacion.com/uploads/pdf/27_esp. $p d f$

Miller, Toby. Global Hollywood 2. Londres: British Film Institute, 2005. ISBN: 9781844570393

Nielsen Online. "Middle agers help Hulu grow 490\%". Marketing charts, 2009.

www.marketingcharts.com/television/middle-agers-helpHulu-grow-490-9125

Netflix. Annual report, 2010.

http://files.shareholder.com/downloads/NFLX/15620367 $21 \times 0 \times 460274 / 17454 c 5 b-3088-48 c 7-957 a-b 5 a 83 a 14 c f 1 b /$ 132054ACL.PDF

Netflix. Q3 2011 Financial statement. http://ir.netflix.com

Parrick, Dave. "Youtube to acquire full-length movies from Sony? From UGC to premium continues". WebTVwire, 2008, 7 April.

www.webtvwire.com/Youtube-to-acquire-full-length-mo viesfrom-Sony-from-ugc-to-premium-continues

Papies, Dominik; Clement, Michael. "Adoption of new movie distribution services on the internet". Journal of media economics, 2008, v. 21, n. 3, pp. 131-157. http://dx.doi.org/10.1080/08997760802300530

Richmond, Will. "Other analysts waking up to concerns about Hulu's business model". VideoNuze, 2009, 20 May. http://www.videonuze.com/article/other-analysts-wakingup-to-concerns-about-hulu-s-business-model

Ryan, Mark-David; Hearn, Gregory N. "Next generation filmmaking': new markets, new methods and new business models". Media international Australia: incorporating culture and policy, 2010, n. 136. http://eprints.qut.edu.au/33169/1/c33169.pdf

Walsh, Mark. "Report: TV networks should be afraid -Very afraid- of Hulu". Media post news, 2009, 14 Sept.

www.mediapost.com/publications/?fa=Articles.showArti cle\&art_aid=113407

Wasko, Janet. How Hollywood works. Londres: SAGE, 2003. ISBN: 9780761968146 